\title{
REPRESENTACIONES SOCIALES DEL CUIDADO DEL ANCIANO EN TRABAJADORES DE SALUD EN UN ANCIANATO
}

Rosalina A.Partezani Rodrigues*

Oséias Guimarães de Andrade**

Sueli Marques***

RODRIGUES, R.A.P.; ANDRADE, O.G. de; MARQUES, S. Representaciones sociales del cuidado del anciano en trabajadores de salud en un ancianato. Rev.latino-am.enfermagem, Ribeirão Preto, v. 9, n. 1, p. 7-12, janeiro 2001.

Este trabajo tiene como objetivo identificar las representaciones sociales que con respecto al cuidado del anciano tiene su cuidador formal en el ancianato. Se trabajó con 15 trabajadores de salud con edades que oscilaron entre 28 y 62 años. Se utilizó como referencial la Teoría de las Representaciones Sociales. La recolección de datos fue realizada a través de la entrevista semi-estructurada y se optó por el análisis de contenido que permitió la elaboración de unas categorías que facilitaran la comprensión de los datos. Dentro de estas están: educación informal, educación formal, satisfacción, tristeza, modos de enfrentamiento y negación.

TÉRMINOS CLAVES: cuidado, cuidadores

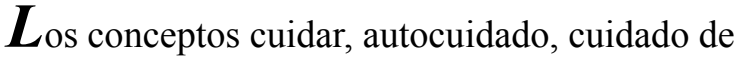
sí, son utilizados largamente en la literatura. Estos conceptos son tan antiguos que el mismo Sócrates, describió el cuidado como el arte de la existencia (FOUCAULT, 1985).

Después del inicio de la existencia del ser humano el termino cuidar paso a hacer parte de la vida cotidiana de este. El cuidar, el autocuidado y el cuidado aún permanecen como conceptos fundamentales para la sobrevivencia del ser humano.

LEININGER (1984) relata la importancia del cuidado en la vida humana, hace referencia al "conocimiento popular" del cuidado, en el cual considera la cultura, los valores, las creencias, los significados, el contexto en que viven los seres humanos, como aspectos que exigen un conocimiento profesional fundamentado en principios científicos.

COLLIÉRE (1989) se refiere al cuidar como un acto indispensable, no solo a la vida humana, sino también, para todo el grupo social, menciona que "el cuidar" es una función primordial a la sobrevivencia de todo el ser vivo.

En la enfermería, los conceptos de cuidar, autocuidado y cuidado de sí, aparecen desde el surgimiento de la enfermería moderna. Varias cuestiones son expresadas en la literatura al respecto del trabajo de las enfermeras, del concepto y valoración de la enfermería y de la relación con las personas que reciben cuidados de enfermería.

HENDERSON (1962), en la década del 50, enfatizó la participación del cliente en el cuidado de sí mismo y definió que la función de la enfermería es prestar asistencia al individuo, sano o enfermo, en el desempeño de las actividades que contribuyen para mantener la salud o para recuperarla, actividades que éste desempeñaría si tuviera fuerza, voluntad y el conocimiento necesario, de tal forma que se logre tornarlo independiente lo más rápido posible.

La práctica del autocuidado consiste en actividades que la persona realiza en su beneficio para mantener la vida, la salud y el bienestar. Es una necesidad de todas las personas, que, si no es satisfecha, puede traer desequilibrios, enfermedad y hasta la muerte.

El hombre, en el transcurso de la vida, aprende a cuidar de sí y de los otros, pero no se puede afirmar que al llegar a la vejez esa habilidad sea preservada. Es importante comprender que ese aprendizaje también

* Profesor Asociado de la Escuela de Enfermería de Ribeirão Preto de la Universidad de São Paulo. Dirección: Av. Bandeirantes, 3900 - Monte Alegre - 14040-902 - Ribeirão Preto - São Paulo - Brasil

**Profesor de la Escuela de Enfermería de Maringá y estudiante de doctorado de la Escuela de Enfermería de Ribeirão Preto de la Universidad de Sâo Paulo, Brasil

***Enfermera y estudiante de doctorado de la Escuela de Enfermería de Ribeirão Preto de la Universidad de São Paulo, Brasil 
puede ocurrir en la vejez, esto quiere decir que no hay edad límite para el ejercicio del autocuidado. Es esencial estar desarrollando constantemente la capacidad para autocuidarse, a lo largo de la vida y aún más, durante la vejez.

El cuerpo del anciano que se tiene que cuidar, ya no es joven ni lleno de vigor, es un cuerpo frágil, menos tónico y con algunas deficiencias, por eso el anciano tiene la necesidad de ser orientado para ser capaz de autocuidarse.

Entre tanto, ocurren diversas situaciones en la vida de los ancianos, tales como la perdida de la familia, recursos económicos insuficientes, conflictos familiares, vivienda inadecuada, que interfieren en el desarrollo de su vida. Una de las alternativas que se observan actualmente son los "hogares residenciales", con el objetivo de ofrecer a los ancianos la posibilidad de una vida digna; es claro que no substituyen la "casa de la familia". Uno de los aspectos que más llama la atención en estas instituciones es que a cada día que pasa los ancianos se van volviendo más frágiles, limitándose así, su capacidad para autocuidarse, llevando entonces a la necesidad de terceros para ayudar a mantener las actividades de su vida diaria.

Considerando que el cuidado es la esencia de la salud y de la enfermería, compete a la enfermera geriátrica mediar en el proceso de cuidar de acuerdo con las necesidades y el contexto en que el anciano vive.

\section{REFERENCIAL TEÓRICO}

La concientización para la práctica de cuidar, cuidar de sí y del autocuidado debe ser rescatada por el profesional del área de la salud, también por los "cuidadores formales****". Con la finalidad de identificar las representaciones sociales que acerca el cuidado del anciano tienen los cuidadores formales que trabajan en un ancianato***** de Ribeirão Preto, SP, se optó por un referencial que facilitara una lectura del concepto que ha sido construido con relación al cuidado del anciano interno, a partir de la visión de aquellos que ejercen el trabajo de cuidarlo. Se utilizó la Teoría de las Representaciones Sociales, pues gracias a ella, se puede abordar de mejor manera el conocimiento adquirido por el sentido común, es decir, el conocimiento espontáneo, ingenuo, entendido como un conocimiento empírico (natural) opuesto al conocimiento científico.
Este conocimiento natural, se constituye a partir de las experiencias y de las informaciones, conocimientos y modelos de pensamiento que recibimos y transmitimos a través de la educación y de la comunicación social (JODELET, 1984).

En este sentido, trabajar en un ancianato, ofrece una gran cantidad de oportunidades y experiencias para aprender a cuidar al anciano. Así mismo, éste aprendizaje permite la estructuración de toda una serie de ideas, creencias y conceptos que los cuidadores formales van desarrollando en relación con el cuidado del anciano, razón por la cual se torna fundamental, rescatar sus opiniones, experiencias, sensaciones y emociones derivadas de su vida cotidiana cuidando del anciano.

Todas estas experiencias se pueden ver reflejadas en las representaciones sociales que estos cuidadores tienen con respecto al envejecimiento y por consiguiente, a las acciones para brindar cuidado en esta fase de la vida. Así entendidas, las representaciones sociales se construyen dentro de los individuos a partir de 2 procesos, la objetivación y el ancoraje (MOSCOVICI, 1978). Con respecto a la primera, esta permite convertir en real un esquema conceptual, es decir, dar consistencia a las ideas y coherencia entre las acciones y las palabras. En relación al segundo, significa la aplicación de ese esquema conceptual a la realidad social y la vida cotidiana, es decir, al desarrollo de nuestros actos y de nuestras actitudes en la sociedad.

Así pues, las representaciones sociales se estructuran con base en 3 dimensiones: la información, la actitud y el campo representacional (imagen); en nuestro caso, la información se relaciona con los conocimientos que los cuidadores tienen acerca del envejecimiento y del anciano a partir de la comunicación compartida socialmente; en cuanto a la actitud, ésta muestra las emociones y la sensibilidad que genera en el cuidador el anciano interno que precisa de cuidado, pudiendo ser favorable, neutra o desfavorable; y por ultimo, el campo representacional se refiere al modelo o imagen que el cuidador hace del anciano.

Este tipo de estudio es importante ya que contribuye a la reflexión sobre la vida de los ancianos que viven separados de sus familias y las implicaciones que esta separación tiene para el desarrollo de su vida. Para ellos, vivir en un ancianato significa pasar a ser dependientes de otras personas quienes se encargan de atender sus necesidades básicas.

Los ancianos como parte de su proceso de

\footnotetext{
**** entendemos como cuidadores formales como una persona vinculada al mercado del trabajo

***** El ancianato del Padre Euclides, es una institución filantrópica con 60 años de funcionamiento y que tiene como objetivo cuidar de los ancianos que no tienen condiciones de autocuidarse
} 
envejecimiento se tornan mas frágiles lo que hace, que ellos necesiten ser cuidados por otras personas. En este sentido, el presente trabajo refleja la práctica diaria de los cuidadores durante su relación interpersonal cuidando a los ancianos.

Así pues, en este estudio, el interés principal es identificar las representaciones sociales elaboradas por los cuidadores formales, con respecto a sus experiencias particulares durante sus actividades cotidianas cuidando a los ancianos.

\section{TRAYECTÓRIA METODOLÓGICA}

Se trata de un estudio exploratorio, descriptivo, realizado en un ancianato filantrópico en la ciudad de Ribeirão Preto, São Paulo. Alberga 93 ancianos de ambos sexos, con edades que varían entre 55 y 92 años. La infraestructura institucional está compuesta por residencias para ancianos físicamente independientes, con cuarto para dos personas y para los ancianos físicamente dependientes, existen cuartos con capacidad para 5 y/o 8 ancianos, los cuales están separados por sexo.

Se optó por entrevistar todos los cuidadores que dan cuidados a los ancianos dependientes (15). Para la recolección de los datos, se utilizó un instrumento (entrevista semi-estructurada) el cual se dividió en 2 partes: en la primera se abordaron datos referentes a los aspectos socio-demográficos que caracterizan los cuidadores tales como edad, sexo, profesión, estado civil, etc. y en la segunda parte, se formularon preguntas abiertas relacionadas con el proceso de cuidado al anciano intentando abarcar aspectos relacionados con la formación recibida, las expectativas personales, los sentimientos particulares, las opiniones y en general su vida cotidiana dentro de la relación de cuidado que establecen a diario con los ancianos.

Las entrevistas se realizaron en la propia institución, con autorización de la Dirección y de las personas entrevistadas, en el mes de diciembre de 1998.

Para el análisis de los datos se utilizó la técnica de análisis temático de contenido, de BARDIN (1991).

\section{RESULTADOS Y DISCUSIÓN}

La población se constituyó de 15 cuidadores, siendo $14(93,3 \%)$ mujeres y $01(6,6 \%)$ hombres, con edades que varían entre 28 y 62 años, con un promedio de 40 años. Con relación a la escolaridad y profesión, 2 $(13,3 \%)$ son técnicas en enfermería y poseen primer grado completo, $2(13,3 \%)$ auxiliares de enfermería, con primer grado completo; $4(26,6 \%)$ son religiosas y $6(40 \%)$ son ayudantes (serviciales), que sin embargo también ejercen el papel de cuidador en el ancianato.

El cuidado de los enfermos, de los niños y de los viejos siempre correspondió a las mujeres; este hecho es histórico y es constante en la mayoría de las sociedades. Tal situación se ve reflejada en esta institución en donde casi la totalidad de los cuidadores son mujeres.

Con base en las declaraciones obtenidas de los entrevistados y teniendo como guía el esquema de análisis propuesto por BARDIN, se pudieron estructurar algunas categorías que permitieron tener una idea general de los aspectos más importantes que conforman las representaciones sociales que los entrevistados han construido en torno al proceso de cuidado del anciano y fueron analizados con base en las declaraciones de los cuidadores del ancianato, las cuales se pueden ser visualizadas en el Cuadro 1.

\section{Cuadro 1 - Distribución de las categorías y sub- categorías de los cuidadores de un ancianato}

\begin{tabular}{|l|l|}
\hline \multirow{2}{*}{ CATEGORIAS } & SUB-CATEGORIAS \\
\hline \multirow{2}{*}{ EDUCACIÓN } & Formal \\
\cline { 2 - 2 } & Informal \\
\hline \multirow{2}{*}{ SENTIMENTOS } & Positivos \\
\cline { 2 - 2 } & Negativos \\
\hline \multirow{2}{*}{ ACTITUDES } & Enfrent amiento \\
\cline { 2 - 2 } & Rechazo \\
\hline \multirow{2}{*}{ VOCACIÓN } & Innata \\
\cline { 2 - 2 } & Desarrollada \\
\hline \multirow{2}{*}{ ACCIONES } & Parcial \\
\cline { 2 - 2 } & Integral \\
\hline
\end{tabular}

La categoría educación comprende dos subcategorías: formal e informal, conforme es observado en la Figura 1.

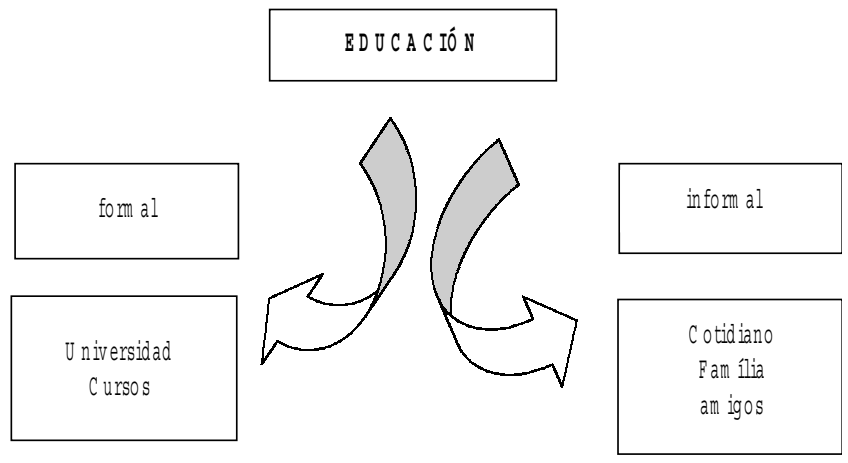

Figura 1 - Educación de los cuidadores del ancianos 
La educación formal es entendida como aquella en que el educando frecuenta un determinado curso o programa, sea en universidades, o en otro curso formal incluyendo uno o varios temas, teniendo en cuenta que sea siempre originario de una institución socialmente legitimada y legalmente constituida. Los cuidadores refirieron que tuvieron oportunidad para el acceso a la educación formal, con el objetivo de mejorar el conocimiento sobre el cuidado con ancianos. Es interesante resaltar que los propios cuidadores fueron los que buscaron esas informaciones. Algunos consideran que participamos de cursos, eventos, encuentros sobre ancianos, pues así, podemos aplicar la teoría en la práctica. Relatan también que en el inicio el cuidado era solo cariño y comprensión sin tener un mayor conocimiento del asunto. Refieren también que la programación diseñada por los cursos frecuentados ofreció la oportunidad para debatir temas sobre la vejez. De los 15 cuidadores, 3 (20\%) refirieron que tuvieron educación formal para cuidar de los ancianos. Entre tanto, para los otros el cuidado es representado como una tarea simple, no existiendo la necesidad de una educación formal.

Los ancianos aislados en esa institución son en su gran mayoría independientes, es decir que tienen capacidad para autocuidarse, por lo tanto, no necesitan de cuidados de alta complejidad. Por otra parte, los cuidadores formales que trabajan en la institución carecen de conocimientos sobre el envejecimiento. La representación social que los cuidadores tienen sobre su trabajo de "cuidar" de los ancianos, es de apoyo y de auto-ayuda.

Se consideró educación informal, aquella recibida en el propio ancianato, con las religiosas a través de las observaciones de los colegas de trabajo y de la comunicación socialmente compartida, como se observa en las unidades de análisis que se encuentran a continuación: aprendí con las hermanas..., ...la colega que trabaja conmigo es quien me enseñó.... ...aprendi viendo a los otros hacer..., ...aprendi en el ejército, los médicos me enseñaron... a calmar los pacientes, a hacer curaciones... aplicar inyecciones y a dar medicamentos, dar amor, dedicación y cariño.

Como se puede observar en los enunciados, los cuidadores reproducen los conocimientos a partir de la práctica cotidiana con los otros cuidadores, la familia y los amigos y con el proprio anciano con quien convive en el cotidiano del cuidado.

La falta de conocimiento formal de los cuidadores cuidar de los ancianos puede ser explicado por la ausencia de una política de preparación de recursos humanos en el área de gerontología y geriatría no solamente en las Universidades sino también en las diversas Instituciones hospitalarias y en los ancianatos.
La categoría sentimientos comprende el conjunto de dos sub-categorías referentes a los sentimientos positivos y negativos de acuerdo con la Figura 2.

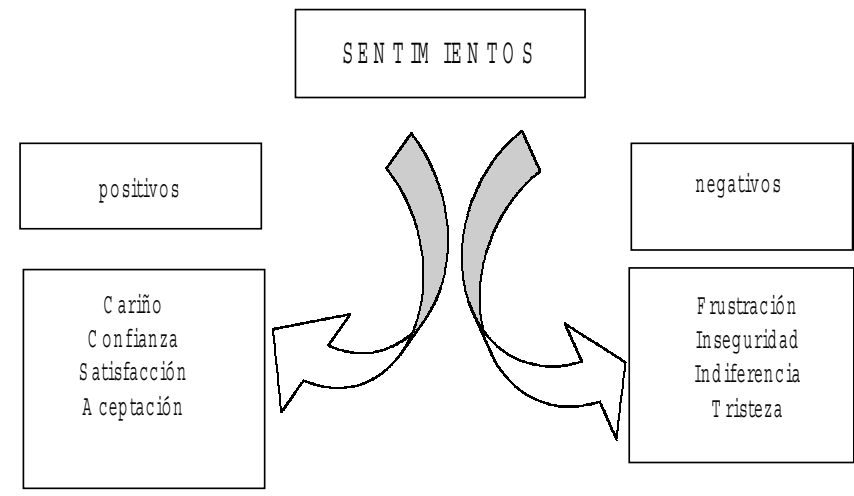

Figura 2 - Sentimientos con respecto al cuidado con el anciano

Podemos observar que el afecto es uno de los elementos simbólicos de las representaciones sociales expresado en esta categoría, de donde emanaron aspectos positivos y negativos. Se incluyen aquí las expresiones ... much a felicidad, congratulada, satisfacción en cuidar de los ancianos..., ... me siento realizada en estar cuidando de alguien, de una persona que necesita de cariño....

De otra forma, miramos que hay contraposiciones en las charlas de los cuidadores tales como ...vacía, triste, deprimente. Es aquí que la mayoría de los papeles sociales terminan, no ofreciendo oportunidad a los ancianos para un nuevo papel. Esta se torna así, una fase monótona de la vida, con pocas o casi ninguna, atribución social. Ese estereotipo es una actitud de la sociedad en general e incorporada por los ancianos y cuidadores, como se puede observar en los enunciados de los cuidadores ... creo solitaria la vejez, es muy triste..., ... la vejez es triste, creo que preciso ayudar a las personas ancianas, voy a estar vieja y también voy a precisar de alguien para ayudarme.

Vivir la vejez, una etapa de la vida en la institución asilar, significa que los proyectos de vida se fueron, la lucha por los ideales y los sueños también se fueron, permaneciendo apenas una persona frágil, dependiente, desanimada es muy dolorosa la vejez, como refiere un cuidador. Ese reflejo de la vejez dependiente, frágil y triste es una imagen del cotidiano de los cuidadores.

Esos sentimientos sean positivos, sean negativos, implican una diversidad de acciones frente a la representación de la práctica de los cuidadores. Los sentimientos positivos evocan motivaciones para cuidar, pero los negativos generan apenas una sensación de cumplimiento de las tareas.

Por otro lado de las actitudes emanaron las siguientes sub-categorías, de acuerdo con la Figura 3. 


\section{A C T IT U D ES}

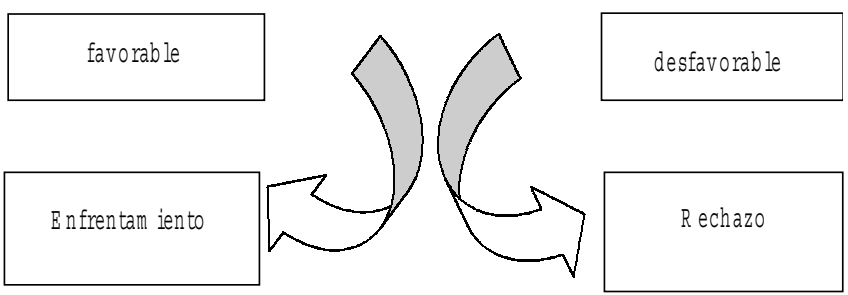

Figura 3 - Actitudes de los cuidadores al cuidar de los ancianos

A dimensión actitud se refiere a la posición que asume el cuidador sea ésta favorable o desfavorable en relación con el cuidado del anciano. A medida que los cuidadores van actuando en el cuidado con los ancianos institucionalizados y más dependientes, estos construyen imágenes de las necesidades que se presentan en el cotidiano. De esa forma, los cuidadores van asimilando los acontecimientos de la vida diaria en la institución, a través de la observación, de las informaciones compartidas entre el equipo actuante y las otras personas que circulan en la institución.

El modo de enfrentamiento también es utilizado por los cuidadores en el cotidiano con los ancianos. Esa sub-categoría es interesante de ser analizada, pasa por el respeto y educación a los mas viejos y frágiles, principalmente por los cuidadores mas jóvenes, de acuerdo con estos ...siento como si ellos (viejos) fueran mis padres. Otro aspecto a ser analizado es que las actividades ludo-terapéuticas como la música, la recreación, la danza y otras técnicas expresivas en que los ancianos participan, también son una forma de amenizar los cuidadores las angustias vividas en su trabajo.

La actitud desfavorable puede ser observada en las unidades de análisis ...no quiero mas trabajar con ancianos, procuro hacer oraciones..., ...viejos frágiles $y$ dependientes dan mucho trabajo para cuidar... BEAUVOIR (1990) describe que cada sociedad crea sus propios valores y actitudes con relación al progreso o rechazo de los hombres y que la vejez debe ser comprendida en su totalidad biológica y cultural.

A través de esa experiencia de cuidar de los ancianos es que los cuidadores proyectan el futuro, y esa proyección esta representada por una vejez dependiente ...tengo que prepararme para no estar viejo así..., ...el cansancio, el estrés del cuidado diario a los ancianos, asociados a los problemas de dificultades de relacionarse debido a las enfermedades de los mismos, tengo que procurar otras alternativas para mi vida, para no tener conflictos.

La vocación, es otra categoría que circula en las palabras de las personas y esta representada en la Figura 4.

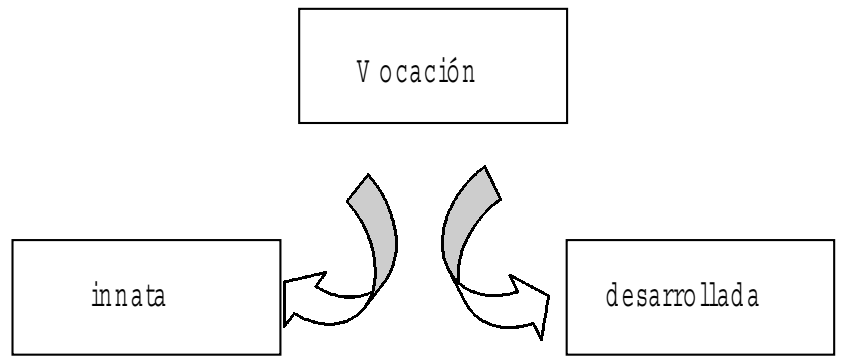

Figura 4 - Vocación de los cuidadores al cuidar de los ancianos

Cuidar a los ancianos requiere también vocación por parte de los cuidadores, pues tratar con estas personas en los momentos de alegría genera satisfacción, aun así, en los momentos de dolor y sufrimiento no es una tarea tan fácil como aparenta ser. Tratar con ancianos dependientes exige de los cuidadores, no solamente vocación innata ya que esta necesita ser complementada con el desarrollo de prácticas, con conocimientos del proceso de envejecimiento y de sus problemas, aceptar la vejez, procurar relacionarse con los ancianos es ofrecer amor. Esta categoría puede ser leída en los enunciados de los cuidadores: ...significa que está dando cuidado para el proprio Cristo, no veo solo una persona, veo el proprio Cristo y cuido de él con amor..., ... las personas dependientes son personas de las cuales todos se apartan para cuidar, es preciso tener paciencia, cariño... en ese momento que los viejos precisan de ayuda... y nosotros tenemos que desarrollar esta tarea.

Las acciones están relacionadas con la existencia de un determinado problema que necesita ser resuelto integral o parcialmente. Esta categoría es presentada en la Figura 5.

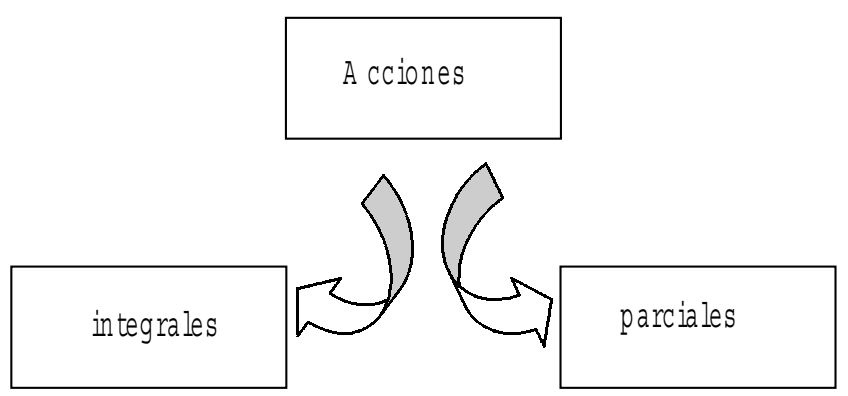

Figura 5 - Acciones desarrolladas en el cuidado del anciano

Las acciones integrales o parciales están relacionadas con las informaciones que los cuidadores tienen en relación con la persona en el proceso de envejecimiento normal o patológico.

En el análisis de las declaraciones dadas por los 
cuidadores a cerca de las actividades cotidianas con los ancianos, pudimos observar que las representaciones están relacionadas con la imagen y la actitud que cada cuidador tiene de manera individual frente al cuidado del anciano. Así, visiones positivas llevan a desarrollar acciones integrales y completas, mientras que visiones negativas generan acciones mas parciales y simples. Lo anterior refuerza el hecho de entender las representaciones sociales como aquellas formas de pensar, sentir y actuar en relación con un objeto (cuidado) y su interdependencia, confirmando la orientación de las representaciones sociales en el comportamiento (JODELET, 1984).

\section{CONSIDERACIONES FINALES}

El trabajo presentando tuvo la finalidad de aplicar la teoría da representación social propuesta por MOSCOVICI (1978). Siendo la representación social una modalidad de conocimiento socialmente elaborada y compartida, con la finalidad principal de construir una dada realidad ligada a un conjunto social (JODELET, 1984); este estudio se orientó en particular, hacia las dimensiones de la representación social.

En relación con la dimensión información, lo que se verificó fue que los cuidadores usan conocimientos basados en principios científicos y también conocimiento popular para prestar el cuidado; por otro lado en el campo representacional, la imagen del anciano es de ...un viejo frágil, dependiente de terceros, que la vejez es una fase de degradación...; y la actitud apareció tanto favorable como desfavorable, muchas veces es de ...procurar otras alternativas para hacer... con mira a propia vejez, y muchas veces acaban teniendo una actitud maternal con los ancianos.

La inquietud de este estudio es de identificar el tipo de producción de conocimientos elaborados por los cuidadores formales del anciano en el ancianato y la influencia que este tipo de producción tiene en la práctica que lleva a cuestiones más profundas sobre la vejez y reflexionar sobre las estrategias para la formación de recursos humanos para lidiar con ese cotidiano tan difícil: del cuidado de ancianos internados dependientes.

Los resultados encontrados muestran que, es necesario hacer una reformulación del modelo tradicional de atención a los ancianos, más sí tenemos en cuenta que, las enfermeras tienen un papel fundamental dentro del proceso de cuidado integral y multiprofesional, el cual es el de integrar y conciliar los intereses y las necesidades tanto de los ancianos, como del equipo de salud y la administración de la institución. Por esto, necesita comprender cual es el significado que el cuidado del anciano tiene para el cuidador.

\section{SOCIAL REPRESENTATIONS OF THE CARE GIVEN TO THE ELDERLY BY HEALTH PROFESSIONALS IN A NURSING HOME}

This study aimed at identifying the social representations of the care given to the elderly by caregivers in a nursing home. Fifteen caregivers aged 28 to 32 years old who worked in a nursing home in the city of Ribeirão Preto, São Paulo, Brazil were subjects for this work and the Theory of Social Representations was used as reference. Data were collected by means of semistructured interviews and analyzed by content analysis, which enabled the elaboration of categories, thus facilitating comprehension. Among such categories are: informal education, formal education, satisfaction, sadness, modes of confrontation and denegation.

KEY WORDS: care, caregivers

\section{REFERENCIAS BIBLIOGRÁFICAS}

01.BARDIN, L. Análise de conteúdo. Lisboa: Edições 70, 1991.

02.BEAUVOIR, S. A velhice. Río de Janeiro: Nova Fronteira, 1990.

03.COLLIÉRE, M. Promover a vida. Trad. de Maria Leonero Braga. Lisboa: Printipo-Damaia, 1989.

04.FOUCAULT, M. História da sexualidade III: o cuidado de si. Rio de Janeiro: Graal, 1985.
05.HENDERSON, V. Princípios básicos para cuidados de enfermagem. Rio de Janeiro: ABEn, 1962.

06.JODELET, D. Représentation sociale: phénomènes, concept et théorie. In: MOSCOVICI, S. Psicologie sociale. Trad. de B. Gonties. Paris: PUF, 1984. p.357-78.

07.LEININGER, M. Care the essence of nursing and health. Thorofare, N.Y: John Wiley, 1984.

08. MOSCOVICI, S. A representação social da psicanálise. Rio de Janeiro: Zahar, 1978. 